\title{
Digital Modulation \& Detection Techniques Using FRFT Basis Functions
}

\author{
C. Vijaya
}

\begin{abstract}
Recently the technological developments in digital modulation techniques have resulted in significant reduction in bit error and computational complexity in their realization. However, researchers are coming up with new techniques to meet the challenges encountered in digital modulation and detection. Amplitude modulation, frequency modulation, phase modulation and also combination of these have been employed to represent / distinguish ones and zeros or even several combinations of groups of ones and zeros of a digital bit stream. At the same time, conventional transmitters and receivers are being replaced with programmable hardware such as general purpose PC / special purpose digital signal processor referred to as dedicated software defined radio (SDR) kit. Except for the antenna section, the entire conventional radio functionality may be replaced with a program developed on special purpose hardware. SDR is thus evolving to be a promising solution for these challenges such as interoperability, global seamless connectivity encountered in wireless communication. While conventional digital modulation techniques such as Quadrature Amplitude Modulation (QAM) and orthogonal frequency division multiplexing (OFDM) employ orthogonal set of basis functions of Fourier Transform, (FT), thought process is going on to take into account dynamic characteristics of the communication channel while developing channel modeling and digital modulation techniques. In this paper an attempt is made to present a digital modulation technique that employ chirp signal which is basis function of Factional Fourier Transform (FRFT) to distinguish various bit combination in digital signal transmission followed by computationally less intensive demodulation technique based on Teager energy measurement.
\end{abstract}

Keywords--- Factional Fourier Transform, Software Defined Radio, Digital Modulation, Teager Energy Operator

\section{INTRODUCTION}

I $\mathrm{N}$ digital communication system, in its simplest form, each bit of message data $0 / 1$ is represented by a signal during the symbol time duration $T_{s}$. The receiver is supposed to detect the signal and take decision whether it is $0 / 1$. Because 0 s and $1 \mathrm{~s}$ are continuous pulses and pulse require infinite band width for its fullest representation, certain band limited signal is transmitted in place of $0 \mathrm{~s}$ and 1s. Multilevel amplitude signaling (PAM) transmits one of $\mathrm{M}$ (referred to as modulation order) signal waveforms for a symbol where a symbol consists of group of $b$ bits. Thus the system will have
$\mathrm{M}=2^{\mathrm{b}}$ different signal waveforms[1]. There are possibilities of one symbol being corrupted by channel noise and being recognized as anothersymbol leading to symbol error or referred to as bit error. To reduce the symbol error orthogonal set of symbols are employed. Correlator at the receiver determines the symbol received. Band width of the communication channel poses yet another constraint in digital modulation system. The limited band width causes each pulse to be represented by fundamental and few of its harmonics (not infinite number of its harmonics) resulting in pulse dispersion. Since pulse corresponding to different symbols spread in time, they interfere with adjacent symbol. This poses problem of intersymbol interference. Instead of pulses, sinusoidal signals with different amplitudes represent the symbol in amplitude shift keying whereas sinusoidal signals with different frequencies represent the symbol in frequency shift keying. Similarly, in phase shift keying, different symbols are represented by signals differing with respect to phases. Coherent and non coherent detection techniques have been developed. The digital modulation techniques sometimes combine together variation in amplitude, frequency and phases in developing a modulation technique. For example, $\mathrm{M}=2^{\mathrm{b}}$-ary Quadrature Amplitude Modulation (QAM) signaling uses waveforms which have different combinations of amplitudes and phases to represent $\mathrm{M}$ different symbols of b bits each. Instead of sending the base band signal using a single carrier, orthogonal frequency division multiplexing (OFDM) transmits signals through multiple carriers. These carriers (also called subcarriers) have different frequencies and they are orthogonal to each other. Orthogonal frequency division multiplexing techniques have been applied in both wired and wireless communications. To reduce the computational complexity in modulation, synchronization, and coherent demodulation, up to 34 subcarriers were usually employed. Orthogonal set of subcarriers derived from basis functions of discrete Fourier Transform (DFT) made it possible to increase the number of subcarriers. The introduction of fast computation of DFT / IDFT made the implementation of OFDM more convenient. In wired systems such as Asymmetric Digital Subscriber Line (ADSL) and high speed DSL OFDM has been employed. In Digital Video Broadcasting (DVB) standards, the number of subcarriers can be more than 8000 and the data rate could go up as high as $15 \mathrm{Mbps}$ [2]. At present, attempts are going on to modify the IEEE 802.16 standard, which may result in an even higher data rate up to $100 \mathrm{Mbps}$. Not only new digital modulation techniques are coming up, there are techniques to distinguish them from each other, which is essential in the case of SDR [3]. 
In the proposed work, digital modulation technique based on Fractional Fourier Transform is presented, which has rotation angle, measured with respect to time axis in the timefrequency plane, as an additional degree of freedom. A demodulation technique based on Teager energy operator is presented. In section 2 and section 3, Factional Fourier Transform and Teager Energy operator are respectively introduced. In section 4 , digital modulation technique using Fractional Fourier Transform and its detection using Teager Energy operator is presented.

\section{FRACTIONAL FOURIER TRANSFORM(FRFT)}

Fourier Transform (FT) is applicable for the analysis stationary signals, whose frequency content does not change with time. As the computation of FT involves inner product of signal and the complex sinusoid basis functions over the entire time duration, local characteristics of the signal are treated as though they are global characteristics [4], due to which FT fails to retain the information about the time duration of occurrence of different frequency components in the original signal. That is, time resolution is zero in FT domain. For nonstationary signal analysis, for example, speech signal, video signal, sun light variations etc., there is a need for a transform, which is a joint function of time and frequency that describes the energy density or signal intensity simultaneously in time and frequency plane. This is referred to as Time Frequency Representation (TFR) [4]. FRactional Fourier Transform (FRFT), Short Time Fourier Transform (STFT) and Wavelet Transform (WT) are examples of linear TFRs. [4]. While STFT is the fundamental TFR, helps in understanding the basics of TFR, has fixed analysis window. It does not catch the rhythm of the signal. WT overcomes the drawback of STFT, in that its basis function, wavelet, can be dynamically scaled and time shifted during analysis. The uniqueness of WT is that there is no unique basis function. Basis function can be selected (Haar, Mexican hat, Morlet, $\mathrm{db} 4$ etc.) based on the characteristics of the signal to be analyzed. Hence it exhibits better time-frequency resolution. FRFT is a special case of WT, with chirp as the basis function [5]. In the proposed work, FRFT is employed for digital modulation.

Commonly used FT can be interpreted as a rotation of time domain signal by an angle $\pi / 2$ in the time-frequency plane and represented as an orthogonal signal representation for sinusoidal signal. A rotation by integer multiple of $\pi / 2$ can thus be defined through repeated application of FT. A rotation angle $\alpha=a \pi / 2$, with $a$ being a real number, leads to a domain that represents the signal both with respect to time and frequency. Signal representation along this intermediate axis making an angle $\alpha$ with time axis, in time-frequency plane has nonzero time and frequency resolutions. As the angle of rotation is fraction $a$ of $\pi / 2$, the transform is known as Fractional Fourier Transform with an order parameter $a$. FRFT serves as an orthonormal signal representation for the chirp signal, a signal whose frequency varies linearly with time. The $a^{\text {th }}$ order FRFT of signal $f(u)$ is a linear operation defined by the integral [5] in (1)

$$
f_{\alpha}(u)=\int_{-\infty}^{\infty} K_{a}\left(u, u^{\prime}\right) f\left(u^{\prime}\right) d u^{\prime}
$$

where

$$
K_{a}\left(u, u^{\prime}\right)=\sqrt{1-j \cot \alpha} e^{j \pi\left(\cot \alpha u^{2}-2 \csc \alpha u u^{\prime}+\cot \alpha u^{2}\right)}
$$

Since computational complexity of FRFT is same as that of FT, it is also a powerful tool in solving many signal processing tasks such as differential equations, optical signal processing, time variant filtering, signal compression [5-7], pattern recognition, digital watermarking, time-frequency signal analysis, Fourier optics and optical information processing etc [5].

\section{TEAGER ENERGY OPERATOR}

Teager Energy Operator (or the Teager-Kaiser Energy Operator), has a small time window, making it ideal for local (time) analysis of signals. It has been derived basically from Lie bracket operator [8] which measures the instantaneous differences in the relative change of rate between two signals $x$ and $y$. Lie bracket operator is defined as in (2)

$$
L[y(t), x(t)]=\dot{x}(t) y(t)-\dot{y}(t) x(t)
$$

If $y(t)=\dot{x}(t)$, then the resulting Lie bracket operator defines Teager Energy Operator and is given by (3)

$$
\psi(x(t))=(\dot{x}(t))^{2}-x(t) \ddot{x}(t)
$$

Here $x(t)$ is the signal with $\dot{x}(t)$ and $\ddot{x}(t)$ being its first and second derivatives respectively. To measure the cross energy between a signal and its higher order derivatives $\mathrm{k}^{\text {th }}$ order Differential Energy Operator (DEO) is also available. Teager Energy Operator is also available in discrete form [8] for analyzing a discrete signal and may be defined as in (4)

$$
\psi(x(n))=x^{2}(n)-x(n-1) x(n+1)
$$

The operator has several applications in signal processing. It is used to determine the energy in the signal. When applied to an AM signal, it results in a signal that resembles envelope of the AM signal, thus, can be used for demodulating AM signal. For example, the amplitude modulated signal in (5) $A_{c} \& \omega_{c}$ are the carrier amplitude and frequency respectively, $m(t)$ is the base band signal and $k$ is the modulation index.

$$
S_{A M}(t)=A_{c}(1+k m(t)) \cos \left(\omega_{c} t\right)
$$

The Teager Energy of AM signal given by (6) and it traces the based band signal, thus demodulates the AM signal. In a similar way Teager Energy demodulates the FM signal.

$$
\psi\left(S_{A M}(t)\right)=A_{c}^{2}(1+k m(t))^{2} \omega_{c}^{2}+\cos ^{2} \omega_{c} t
$$

Teager Energy Operator, being a non-linear operator has been used in speech processing. For example, nonlinear process of producing speech signals can be visualized as combination of AM and FM signal since typical speech signal has amplitude variations as well as frequency variations throughout the time domain. Thus speech is referred to as AM-FM type of signal. AM-FM modulation model for speech signal represents a speech resonance formant as an AM-FM 
signal. Each resonance is demodulated into instantaneous amplitude and frequency signals using the energy separation algorithm (ESA) based on energy operator. Speech synthesis is possible by reconstructing the formant bands from the amplitude and phase signals as obtained by applying ESA. The same model is assumed in speech recognition also [9].

Proposed Digital Modulation and Detection: In reality, communication channel is not static. To model the nonstationary characteristic of the real channel, time frequency representation (TFR) methods have to be exploited which may be with or without any additional computational complexity. In the proposed work, bit stream to be transmitted is grouped into b bits. Basis functions of FRFT are used for representing each $b$ bit combination in the bit stream. Each group of b bits is represented by / distinguished by chirp basis function with different chirp rate. These basis functions are orthogonal. Number of different chirp basis functions $\mathbf{M}$ required depends on the number of ' $b$ ' bits in each group, thus, $\mathrm{M}=2^{\mathrm{b}}$.

\section{PROpOsed Digital Modulation: FRM}

Since by changing the rotation angle of FRFT, different orthogonal basis functions are generated, and the same are used in proposed digital modulation, the technique may be referred to as FRFT rotation angle modulation, FRM. A group of ' $b$ ' bits each is represented by $f_{\alpha, n}(t)$ given in equation (7) with a particular rotation angle. The signal that is transmitted will be $f_{\alpha, n}(t)$, rotation angle being dependent on the bit combination. The function $f_{\alpha, n}(t)$ is derived in such a way that in Fractional Fourier domain it corresponds to an impulse [10]. In this respect, it is similar to a sinusoid being an impulse in Fourier domain. Thus,

$$
\begin{gathered}
f_{a, n}(t)=\sqrt{(\sin \alpha+j \cos \alpha) / T} e^{\left(-j \frac{t^{2}+(2 n \pi \sin \alpha / T)^{2}}{2} \cot \alpha+j 2 n \pi t / T\right)} \\
\text { for }-T / 2 \leq t \leq T / 2
\end{gathered}
$$

The frequency of $\mathrm{n}^{\text {th }}$ basis function [10], $f_{\alpha, n}(t)$ is given by (8)

$$
\omega_{\alpha, n}=\frac{2}{T}(2 n \pi / T-t \cot \alpha)
$$

For $n=10$ and for three different rotation angles, 0.033pi, $0.055 \mathrm{pi}$ and $0.0767 \mathrm{pi}$ radians, plots of $f_{\alpha, n}(t)$ are shown in figure 1. Theoretically, infinite number of basis functions can be generated with minute difference in rotation angle \&/or $n$ value. However, challenge is that detection algorithm should be able to distinguish the basis function or the rotation angle (or chirp rate) and hence should be able to detect the bit combination. In the present work, $b=5$ bits, rotation angle ranges from $20^{0}$ to $51^{0}$, with $20^{\circ}$ representing $00000,21^{0}$ representing 00001 and so on.

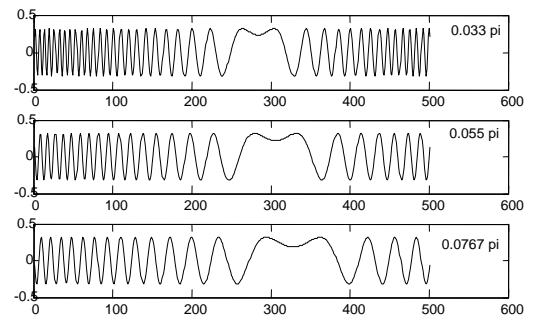

Figure1: Basis Functions for 3 Different Rotation Angles

\section{PROPOSED FRM DETECTION}

Correlation between all the possible basis functions and the one received may be computed. The chirp rate of the basis function exhibiting maximum correlation with received basis function can then determine the bit combination received. But it is computationally intensive process. Possibility of use of Teager energy operator extended to cover complex signals has been exploited in the proposed work to determine the rotation angle of the basis function and hence the bit combination represented. Teager energy is also sum of the energy of the real and imaginary parts of the signal $[11,12]$. Teager energy operator for complex signal $\mathrm{x}(\mathrm{t})$ is given by (9)

$$
\psi(x(t))=\dot{x}(t) \dot{x}^{*}(t)-\frac{1}{2}\left(x^{*}(t) \ddot{x}(t)+x(t) \ddot{x}^{*}(t)\right)
$$

Applying the same to the basis function in (7),

$$
\begin{gathered}
\psi\left(f_{\alpha, n}(t)\right)=\dot{f}_{\alpha, n}(t)\left(\dot{f}_{\alpha, n}^{*}\right)(t)-\frac{1}{2}\left(f_{\alpha, n}^{*}(t) \ddot{f}_{\alpha, n}(t)+f_{\alpha, n}(t) \ddot{f}_{\alpha, n}^{*}(t)\right) \\
\dot{f}_{\alpha, n}(t)=j f_{\alpha, n}(t)(2 n \pi / T-t \cot \alpha) \\
\ddot{f}_{\alpha, n}(t)=-j f_{\alpha, n}(t) \cot \alpha+j(2 n \pi / T-t \cot \alpha) \dot{f}_{\alpha, n}(t) \\
\therefore \psi\left(f_{\alpha, n}(t)\right)=\frac{2}{T}(2 n \pi / T-t \cot \alpha)^{2}=\omega_{\alpha, n}^{2}
\end{gathered}
$$

Thus it is found to be equal to the square of the frequency of the $n^{\text {th }}$ basis function, minima of which occurs at different points in time depending on rotation angle. This characteristic of Teager energy of $f_{\alpha, n}(t)$ identifies the basis function and hence the corresponding bit combination represented.

\section{RESUlts AND DisCUSSION}

The plot of 32 different Teager energy plots for rotation angle differing by 0.0056 pi radians $\left(1^{0}\right)$ is shown in figure 2 . It is observed that these Teager energy plots differ at the point in time of occurrence of their respective minimum peak. To differentiate better, line of reference along y axis is chosen to be slightly above and the same plot is shown in fig. 3A. Further, the part of the plot now crossing zeros is zoomed and shown in fig. 3B. The point in time where energy crosses zero is noted. The plot of points in time of zero crossings with respect to rotation angle is shown in fig. 4. The same data is given in table 1 . Since the points of zero crossings are unique, they identify the basis function and thus detection of combination of $b$ bits. Teager energy of the received signal is computed and the point of zero crossings is found. The corresponding $\mathrm{b}$ bits combination is found by referring to table 
1. For example, if zero crossing is found to be at $t=132$, the corresponding rotation angle is $31^{\circ}$. The bit combination represented by $31^{0}$ is 01100 .

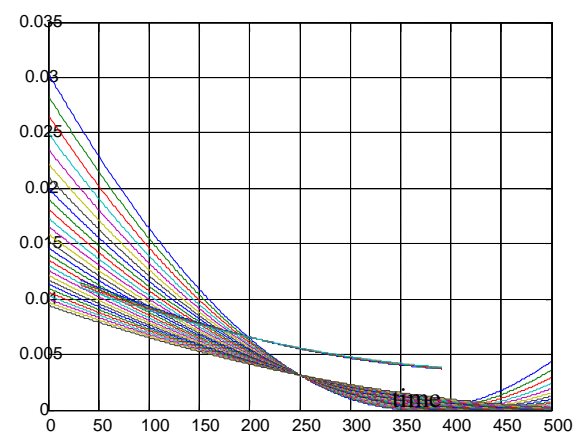

Figure 2: 32 Different Teager

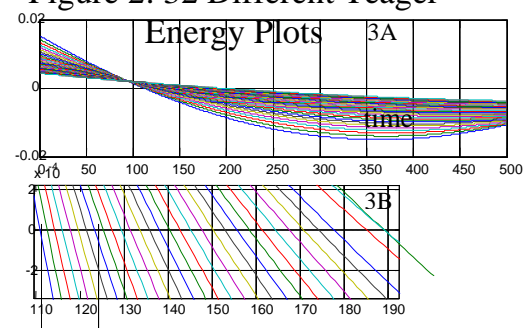

Figure 3: ALine of Reference Shifted Up, 3B. Plots crossing zeros Zoomed

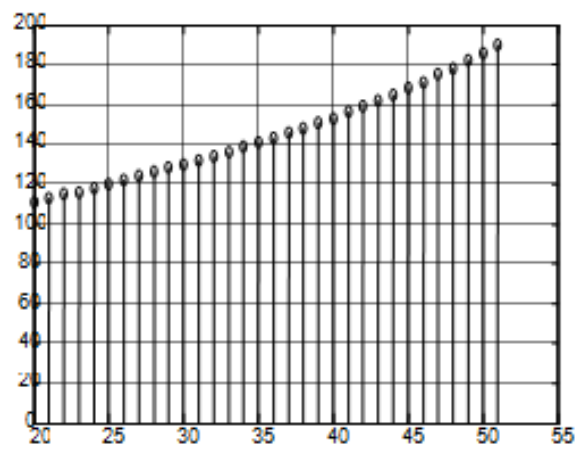

Figure 4: Points in Time of Zero Crossing

Table 1: Rotation Angle (degrees) \& its Identification on Time Axis (Plot in fig.4)

\begin{tabular}{|l|l|l|l|l|l|l|l|l|}
\hline Rotation angle & 20 & 21 & 22 & 23 & 24 & 25 & 26 & 27 \\
\hline $\begin{array}{l}1^{\text {st }} \text { zero cross in } \\
\text { fig.3B }\end{array}$ & 111 & 113 & 115 & 116 & 118 & 120 & 122 & 124 \\
\hline Rotation angle & 28 & 29 & 30 & 31 & 32 & 33 & 34 & 35 \\
\hline $\begin{array}{l}1^{\text {st }} \text { zero cross in } \\
\text { fig.3B }\end{array}$ & 126 & 128 & 130 & 132 & 134 & 136 & 139 & 141 \\
\hline Rotation angle & 36 & 37 & 38 & 39 & 40 & 41 & 42 & 43 \\
\hline $\begin{array}{l}1^{\text {st }} \text { zero cross in } \\
\text { fig.3B }\end{array}$ & 143 & 146 & 148 & 151 & 153 & 156 & 159 & 162 \\
\hline Rotation angle & 44 & 45 & 46 & 47 & 48 & 49 & 50 & 51 \\
\hline $\begin{array}{l}1^{\text {st }} \text { zero cross in } \\
\text { fig.3B }\end{array}$ & 165 & 168 & 171 & 175 & 178 & 182 & 186 & 190 \\
\hline
\end{tabular}

\section{CONCLUSION}

A method of digital modulation, FRM and its detection is explained. While modulation selects one of the orthogonal functions with certain rotation angle for basis function of
FRFT for representing / transmitting b bits, the detection is based on measurement of its Teager energy. Since computational complexity of FRFT is same as that of FT and detection involves Teager energy measurement which is computationally less intensive than computing correlation or inverse FRFT, the proposed method is computationally comparable with that of methods involving FT. In the proposed method it is the resolution with which the Teager energies can be distinguished, for any basis function, decides the upper limit on b bits.

\section{ACKNOWLEDGMENT}

The author expresses her sincere thanks to the Principal, SDMCET, Dharwad and HOD, Department of ECE, SDMCET, Dharwad, for encouraging the work and accepting the paper for presentation.

\section{REFERENCES}

[1] W.Y. Yang, Y.S. Cho, W.G.Jeon, J.W. Lee, J.H. Paik, J. Kim, M. Hyun Lee, K.I. Lee, K.W. Park, K.S. Woo, "MATLAB Simulink for Digital Communication”, $2^{\text {nd }}$ Edition Hongrung Publishing, Korea, 2012.

[2] Y. Xiao, "Orthogonal frequency division multiplexing modulation and inter-carrier interference cancellation”, (Doctoral dissertation, Institute of Automation, 2003.

[3] S. Salman and C. Vijaya, "Algorithm for Distinguishing Modulation Techniques for SDR", Conference on Advancements in Communication \& Computing Systems, 2012.

[4] L. Cohen, "Time-frequency distributions-a review", Proceedings of the IEEE, Vol.77, No.7, Pp.941-981, 1989.

[5] H.M. Ozaktas, Z. Zalevsky and M.A. Kutay, "The fractional Fourier transform”, Pp. 4-6, 2001.

[6] C. Vijaya and J.S. Bhat, "Signal compression Based on Discrete Fractional Fourier Transform and SPIHT", Elsevier Science Publications, Signal Processing, Vol.86, Pp. 1976-1983, Aug. 2006

[7] C. Vijaya and J.S. Bhat, "Sliding discrete fractional transforms", Signal Processing, Vol.88, Pp.247-254, 2008.

[8] University of Oslo, Department of Informatics, "Signal processing using the Teager Energy Operator and other nonlinear operators", Eivind Kvedalen, 2003.

[9] P. Maragos, J.F. Kaiser and T.F. Quatieri, "Energy Separation in Signal Modulations with Application to Speech Analysis", IEEE Transactions Signal Processing, Vol.41, Pp.3024-3051, 1993.

[10] M. Martone, "A multicarrier system based on the fractional Fourier transform for time-frequency-selective channels", IEEE Transactions on Communications, Vol.49, No.6, Pp.1011-1020, 2001.

[11] P. Margos and A.C. Bovik, "Image Demodulation Using Multidimensional Energy Separation”, Journal of the Optical Society of America, Vol.12, Pp. 1867-1876, 1995.

[12] R. Hamila, J. Astola, F. Alaya Cheikh, M. Gabbouj, and M. Renfors, “Teager Energy and the Ambiguity Function", IEEE Transactions on Signal Processing, Vol.47, Pp. 260-262, 1999.

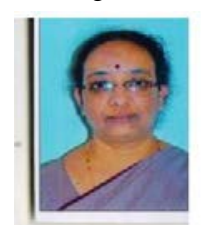

C. Vijaya received her BE (ECE) degree from Karnataka university Dharwad, M. Tech (Digital Electronics \& Communication Systems) degree from Mysore University and $\mathrm{PhD}$ Degree from Karnataka university Dharwad. At present she is working as Professor in the Department of Electronics \& Communication Engineering in SDM College of Engineering \& Technology, Dharwad, India. She is member of ISTE. She has authored a book on Digital Signal Processing. She has to her credits papers published on applications of Fractional Fourier Transform in Elsevier Signal Processing and in National and International Conferences. Her areas of interests are signal processing, cryptography, nano signal processing and software defined radio. 